\title{
Reproducibility and reliability of the occlusal sketch
}

Inter- and intra-operator reliability of the recording of occlusal contacts using 'occlusal sketch' acetate technique S. J. Davies, R. J. M. Gray, M. Z. Al-Ani, P. Sloan and H. Worthington Br Dent J 2002; 193: 397-400

Objectives

To develop a simple way of recording occlusal contacts with proven inter- and intra-operator reliability.

\section{Setting}

Clinical skills laboratory in the University Dental Hospital of Manchester.

Materials and methods

The marked static occlusal contacts of 20 sets of models were recorded in a pseudo-clinical situation, by three dentists and in addition by one dentist on two occasions using a schematic representation of the dental arch - the 'occlusal sketch'.

Results

The median of Kappa agreement for inter- and intra-operator reliability was almost perfect.

\section{Conclusions}

The occlusal sketch is a simple, inexpensive and easy way of recording the results of an occlusal examination using marking papers.

\section{IN BRIEF}

- 'Occlusal sketch' is a simple and inexpensive technique for recording occlusal contacts.

- The 'occlusal sketch' shows a high degree of inter- and intraoperator reliability.

- This technique could be useful for researchers who wish to quantify occlusal changes during different treatment modalities.

- 'Occlusal sketch' can be used, by practising dentists, as a permanent record of the patient's occlusion.

\section{COMMENT}

When it comes to the survival of the restorations we place, controlling occlusal forces is probably crucial. I say 'probably' because the evidence for the impact of occlusion on restorations, or for that matter of restorations on the occlusion, is limited. It is not that this assertion is not likely to be true (it is), but the lack of evidence and consequently of understanding is largely a result of the difficulty in measuring the position of occlusal contacts accurately. This short paper is a step in the right direction. Although the contacts recorded were limited to intercuspal contacts, the principles could equally apply to guidance teeth. It describes a geometric approach to recording contacts. Far from being a simple 'how to do it' article, it successfully demonstrates that the technique is reproducible within and between clinicians.

A proven method for recording occlusal contacts has both clinical and scientific value. As a clinician with a complex case to send to the laboratory, accurate mounting of casts is critical, saving time and money at final fitting. Whilst an occlusal record like that described here may not often be necessary, its reproducibility might make it a useful adjunct in particularly difficult cases. However, it is in clinical trials of restorations that this method may find greatest use. Researchers need to make a confident and reproducible record of the positions of occlusal contacts so that the impact of occlusion on restorations can be included as a factor in the analysis of survival and failure. To date this has rarely, if ever, been done. Anyone who has ever used a Kappa score to try to measure inter-examiner variability will know just how good a score of over 0.8 is, it represents a very high level of agreement. This technique can be used with confidence - at least with mounted casts.

A number of questions remain to be answered. The occlusal sketches showed in this paper illustrate a rather idealised occlusion. Real patients often have less regular arches and it remains to be clarified just how straightforward, accurate and reproducible the records are in these cases. Furthermore, the testing was undertaken on marked casts. There is no reason why natural teeth cannot be recorded in the same way, but the simple act of marking the teeth is substantially more difficult with a mouth full of saliva. Nevertheless this represents a starting point for a scientific and consistent approach to recording occlusion.

Dr J. G. Steele, Senior Lecturer in Restorative Dentistry, School of Dental Sciences, Newcastle 